\title{
Olanzapine-Induced Weight Gain and Increased Visceral Adiposity is Blocked by Melatonin Replacement Therapy in Rats
}

\author{
Murray A Raskind ${ }^{1,2}$, Brianna L Burke², Norman J Crites², Andre M Tapp ${ }^{1,2}$ and Dennis D Rasmussen*,1,2 \\ 'VA Puget Sound Health Care System, Mental IIIness Research, Education and Clinical Center, Seattle, WA, USA; ${ }^{2}$ Department of Psychiatry and \\ Behavioral Sciences, University of Washington, Seattle, WA, USA
}

\begin{abstract}
The atypical antipsychotic drug olanzapine increases body weight and visceral adiposity in schizophrenia. In rats, aging-associated increased body weight and visceral adiposity are reversed by administration of the pineal hormone melatonin. We asked if melatonin similarly would reverse olanzapine-induced increased weight and visceral adiposity in rats. Four groups ( $n=1 \mathrm{I} / \mathrm{group})$ of female rats (240-250 g) were treated for 8 weeks with olanzapine, melatonin, olanzapine + melatonin, or vehicle alone in drinking water. Body weight and food and water consumption were determined weekly, locomotor activity at weeks 3 and 6, and nocturnal plasma melatonin concentration at week 7. At week 8 , the rats were killed and visceral (perirenal, retroperitoneal, omental, and mesenteric) fat pads dissected and weighed. Olanzapine treatment reduced nocturnal plasma melatonin by $55 \%(p<0.00 \mathrm{I})$, which was restored to control levels by olanzapine + melatonin. Body weight increased 18\% in rats treated with olanzapine alone, but only 10\% with olanzapine + melatonin, $5 \%$ with melatonin alone, and $7 \%$ with vehicle control. Body weight and visceral fat pad weight increases in rats treated with olanzapine alone were greater than in each of the other three groups (all $p<0.01$ ), which were not significantly different. These results suggest that olanzapine-induced increases in body weight and visceral adiposity may be at least in part secondary to olanzapine-induced reduction of plasma melatonin levels, and that melatonin may be useful for the management of olanzapine-induced weight gain in humans.
\end{abstract}

Neuropsychopharmacology (2007) 32, 284-288. doi:I 0.1038/sj.npp. I 301093; published online 10 May 2006

Keywords: olanzapine; obesity; melatonin; schizophrenia; antipsychotic drug

\section{INTRODUCTION}

The atypical antipsychotic drugs ('atypicals') have become the drugs of choice for treatment of schizophrenia and other chronic psychotic disorders, largely because of their low incidence of extrapyramidal adverse effects (Leucht et al, 1999). Unfortunately, some of the most widely used atypical antipsychotics frequently induce substantial weight gain and increased visceral adiposity (American Diabetes Association, 2004; Wirshing et al, 1999). These metabolic adverse effects are associated with hyperglycemia and dyslipidemia, and increase long-term risk for diabetes mellitus, ischemic heart disease, and overall mortality (Fontaine et al, 2001; Krotkiewski et al, 1983; Kissebah et al, 1982). They also compromise compliance with long-

* Correspondence: Dr DD Rasmussen, VA Puget Sound Health Care System, II6MIRECC, 1660 S Columbian Way, Seattle, WA 98108, USA, Tel: + I 206277 3370, Fax: + I 206768 5456, E-mail: drasmuss@u.washington.edu

Received 29 November 2005; revised I 4 February 2006; accepted 16 February 2006 term treatment regimens and quality of life (Allison $e t$ al, 2003).

Among the commonly prescribed atypicals, olanzapine has been reported to have the greatest propensity to produce weight gain (Gothelf et al, 2002; Kinon et al, 2001; Wirshing et al, 1999). In the recent Clinical Antipsychotic Trials of Intervention Effectiveness (CATIE) study initiated by the NIMH to compare effectiveness of antipsychotic drugs, olanzapine appeared to be more effective than the other drugs studies, but it was associated with greater weight gain and other metabolic changes implicated in the development of metabolic syndrome (Lieberman et al, 2005). Although the negative public health implications of the weight gain and other metabolic adverse effects of olanzapine have become a matter of concern to both the psychiatry and endocrinology communities (American Diabetes Association, 2004), olanzapine continues to be widely prescribed. Approaches to reducing atypical-induced weight gain in schizophrenia using diet, exercise, and appetite suppressant drugs have demonstrated some short-term benefits (Henderson et al, 2005; Graham et al, 2005a, b). However, long-term efficacy of such 
approaches to maintaining weight reduction is unlikely given disappointing results of such approaches to long-term weight reduction in the general population (Glazer, 2001). Consequently, finding an effective intervention that would prevent or reverse weight gain induced by olanzapine is an important goal.

A potential approach to preventing atypical-induced weight gain is suggested by our previous studies with the hormone melatonin. Melatonin is secreted nocturnally by the pineal gland into blood and, at least in some species, into cerebrospinal fluid (reviewed in Cagnacci, 1996). Although the best understood function of melatonin is photoperiodic entrainment of endogenous circadian rhythms, melatonin also has a role in regulating energy balance and fat distribution in at least some species (Nelson and Demas, 1997). Pinealectomy, which decreases but does not eliminate circulating melatonin, has been demonstrated to increase body weight in rats (Peschke et al, 1987). Because normal aging in humans and rats is associated with both increased visceral adiposity and decreased circulating melatonin concentrations (Grad and Rozencwaig, 1993), we previously investigated the effects of daily melatonin supplementation on aging-associated increased body weight and visceral fat in middle-aged rats. Melatonin treatment reduced body weight, and visceral fat was restored to youthful levels within 10 weeks (Rasmussen et al, 1999, 2001; Wolden-Hanson et al, 2000). Continued melatonin supplementation until old age maintained suppression of this visceral fat accumulation (Rasmussen et al, 1999). We have now asked if olanzapine increases body weight and visceral adiposity in rats and, if so, whether melatonin replacement likewise reverses these changes. We also asked if olanzapine suppresses plasma melatonin concentrations.

\section{MATERIALS AND METHODS}

Forty-four female Sprague Dawley rats weighing 240-250 g at the start of treatment were individually housed with a $12 \mathrm{~h} / 12 \mathrm{~h}$ light/dark cycle. Chow and water were available ad libitum throughout the study. All procedures were approved by the University of Washington Institutional Animal Care Committee and the protocol was in accord with the NIH guide for Care and Use of Laboratory Animals.

Four treatment groups of 11 rats each received olanzapine, melatonin, olanzapine + melatonin, or vehicle alone in their drinking water for 8 weeks. Details of olanzapine solution preparation have been described previously (Gao et al, 1998). The olanzapine concentration in the drinking water was initially $0.033 \mathrm{mg} / \mathrm{ml}$; this concentration was modified according to measured daily water intake and body weight during the course of the experiment to maintain a dosage of approximately $2 \mathrm{mg} / \mathrm{kg} /$ day. This olanzapine dose and method of administration have been demonstrated to maintain rat plasma olanzapine levels in the human therapeutic range (Gao et al, 1998). Melatonin was dissolved in $100 \%$ ethanol and added to the drinking water at a final concentration of $0.4 \mu \mathrm{g} / \mathrm{ml}$. All water bottles containing melatonin were covered with aluminum foil to prevent melatonin photodegradation. The final ethanol (vehicle) concentration was $0.01 \%$ for all treatment groups. Fresh solutions were prepared twice weekly.
Body weights were determined weekly. Food and water consumption were determined over each weekend. Twelvehour nocturnal locomotor activity was measured by infrared beam breaks at approximately week 3 of treatment and again at approximately week 6 using an Opto-Varimax Mini Animal Activity Monitoring System (Columbus Instruments, Columbus, OH). Tail blood samples for melatonin assay were collected at the midpoint of the dark period in week 7 , briefly exposing each rat to dim red light. After 8 weeks of treatment, rats were killed by decapitation during the last hour of the light period. The retroperitoneal, perirenal, omental, mesenteric, and ovarian fat pads were dissected and immediately weighed.

Plasma melatonin was measured by radioimmunoassay using a Melatonin Rat RIA kit from Labor Diagnostika Nord Gmbh \& Co., KG (Nordhorn, Germany). All samples were analyzed in a single assay, with $7 \%$ intra-assay coefficient of variation.

Data were evaluated by analysis of variance followed by post hoc Student-Newman-Keuls multiple comparison tests. Data are presented as means \pm SEM; $p<0.05$ was considered significant.

\section{RESULTS}

After 8 weeks of treatment, body weight differed significantly among groups $(\mathrm{F}=8.8, p<0.001)$, with higher weight in the olanzapine group than in each of the other groups (all $p<0.001$ ), which were not significantly different (Figure 1 ). Body weight of the olanzapine-treated rats increased $17.7 \pm 1.8 \%$ over 8 weeks; in contrast, the body weights of rats receiving olanzapine + melatonin, melatonin alone, or vehicle alone increased only $9.8 \pm 1.9,4.9 \pm 1.6$, and $7.3 \pm 1.7 \%$, respectively. Percent weight gain in olanzapine-treated rats was significantly greater than in each of the other three groups (all $p<0.01$ ), which were not significantly different. Total visceral (perirenal + retroperitoneal + omental + mesenteric) fat pad weights were

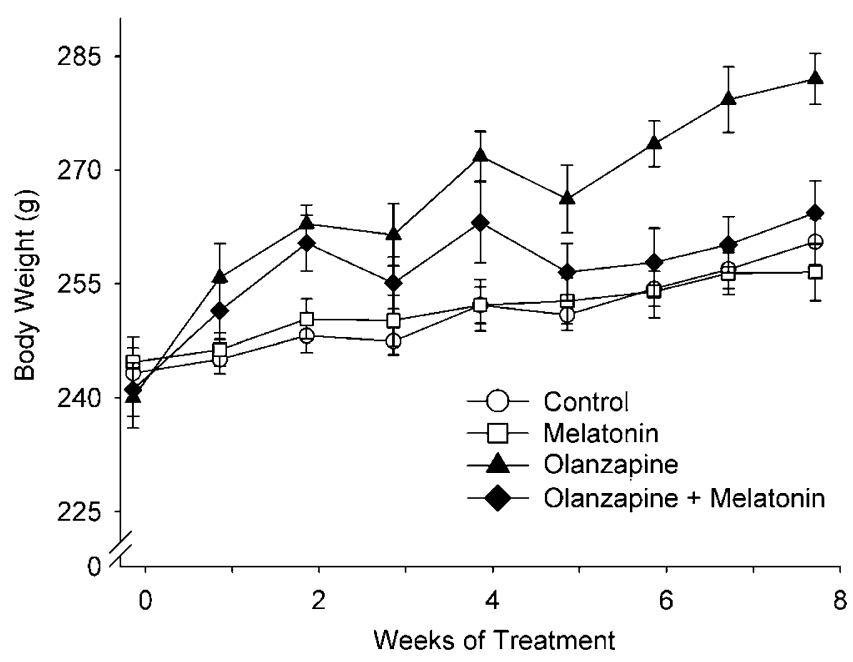

Figure I Body weight change throughout the course of the study. Each data point represents the mean \pm SE of I I rats. At week 8 of treatment, body weight was increased $(p<0.00 \mathrm{I})$ in olanzapine-treated rats relative to each of the other treatment groups. 
increased approximately $37 \%(p<0.001)$ by olanzapine and restored to control levels by olanzapine + melatonin (Figure 2). This pattern was also apparent for each of the individual visceral fat pads (Table 1). The large intraabdominal ovarian fat pad exhibited a similar pattern of weight changes, with an increase in response to olanzapine $(2.8 \pm 0.1$ vs $2.0 \pm 0.1 \mathrm{~g}$ in the vehicle control-treated rats, $p<0.01)$ but no significant change in response to olanzapine + melatonin $(2.4 \pm 0.2 \mathrm{~g})$ or melatonin treatment alone $(1.7 \pm 0.1 \mathrm{~g})$.

Nocturnal plasma melatonin levels in week 7 for rats receiving control, melatonin, olanzapine, and olanzapine + melatonin treatments were $197 \pm 24,519 \pm 57,88 \pm 15$, and $198 \pm 31 \mathrm{pg} / \mathrm{ml}$, respectively. Consequently, nocturnal plasma melatonin concentrations in week 7 of treatment were suppressed $55 \%(p<0.001)$ in the olanzapine-treated rats, relative to control treatment, and these nocturnal plasma melatonin levels were restored to normal by olanzapine + melatonin treatment.

Nocturnal ambulatory activity was determined as total sequential infrared beam breaks per $12 \mathrm{~h}$ night for each rat on each of two nights, after approximately 3 and 6 weeks of treatment. As the activities were not significantly different

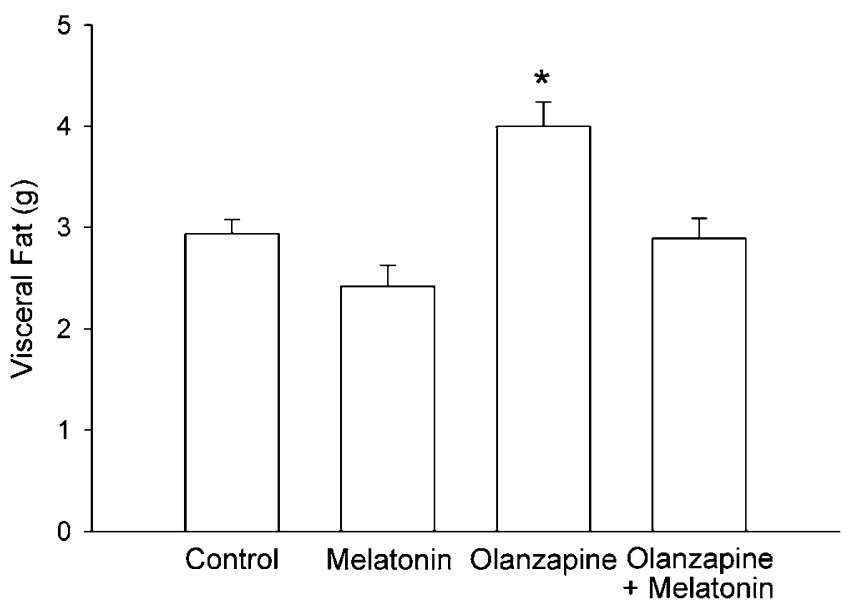

Figure 2 Visceral fat weight determined by dissection at completion of the study. Visceral fat weight for each rat was calculated as the sum of the mesenteric, omental, perirenal, and retroperitoneal fat pad weights. Data represent the mean \pm SE of I I rats/treatment group. $* p<0.00$ I vs all other groups. between the two determinations, the average of the two values for each rat was used for analysis. The average beam breaks/night for the rats receiving control, melatonin, olanzapine, or olanzapine + melatonin treatment were $11740 \pm 430, \quad 10945 \pm 529, \quad 7946 \pm 364$, and $8068 \pm 331$, respectively. Consequently, nocturnal locomotor activity was decreased $32 \%(p<0.001)$ by olanzapine and addition of melatonin treatment did not alter this decrease.

There was evidence of an initial 2-week increase in food consumption in the olanzapine-treated rats, which was not altered by addition of melatonin treatment (Table 2). Food consumption during the remaining 6 weeks of the study was not significantly different among the treatments (Table 2).

\section{DISCUSSION}

Olanzapine treatment for 8 weeks increased body weight and visceral adiposity in adult female rats. These responses to olanzapine in rats are similar to those observed in persons with schizophrenia or other psychiatric disorders treated with olanzapine (Wirshing et al, 1999; Stein et al, 2002). The olanzapine-induced increases in rat body weight and visceral adiposity were associated with olanzapineinduced decreases in nocturnal plasma melatonin levels. Daily oral melatonin replacement sufficient to return nocturnal plasma melatonin levels to normal also reversed body weight and visceral adiposity to normal. This suggests that olanzapine-induced increases in body weight and visceral adiposity may be at least in part secondary to olanzapine-induced changes in melatonin secretion.

Table 2 Average Daily Food Consumption

\begin{tabular}{lcc}
\hline & $\begin{array}{c}\text { During first 2 } \\
\text { weeks of } \\
\text { treatment (g/day) }\end{array}$ & $\begin{array}{c}\text { During subsequent } \\
\mathbf{6} \text { weeks of } \\
\text { treatment (g/day) }\end{array}$ \\
\hline Control & $19.8 \pm 0.4$ & $19.4 \pm 0.3$ \\
Melatonin & $19.9 \pm 0.3$ & $19.4 \pm 0.3$ \\
Olanzapine & $21.8 \pm 0.3^{\mathrm{a}}$ & $20.0 \pm 0.3$ \\
Olanzapine+melatonin & $21.6 \pm 0.4^{\mathrm{a}}$ & $19.6 \pm 0.3$ \\
\hline
\end{tabular}

Data represent mean \pm SE of I I rats/treatment group.

$a_{p}<0.01$ vs control and melatonin.

Table I Visceral Fat Pad Weights

\begin{tabular}{lcccc}
\hline & Perirenal (g) & Retroperitoneal (g) & Omental (g) & Mesenteric (g) \\
\hline Control & $0.60 \pm 0.02$ & $0.66 \pm 0.06$ & $0.38 \pm 0.02$ & $1.30 \pm 0.10$ \\
Melatonin & $0.43 \pm 0.06$ & $0.57 \pm 0.07$ & $0.33 \pm 0.04$ & $1.09 \pm 0.08$ \\
Olanzapine & $0.93 \pm 0.06^{\mathrm{a}}$ & $0.83 \pm 0.04^{\mathrm{b}}$ & $0.52 \pm 0.04^{\mathrm{c}}$ & $1.72 \pm 0.15^{\mathrm{d}}$ \\
Olanzapine+melatonin & $0.58 \pm 0.05$ & $0.65 \pm 0.06$ & $0.44 \pm 0.04$ & $1.22 \pm 0.08$ \\
\hline
\end{tabular}

Weights of each individual fat pad were compared between treatments by one-way analysis of variance with Student-Newman-Keuls multiple comparisons without Bonferroni correction, to reveal consistency of patterns among fat pads.

${ }^{a} p<0.00$ I vs all other groups.

${ }^{b} p<0.05$ vs melatonin.

$c_{p}<0.0$ I vs melatonin, $p<0.05$ vs control.

$\mathrm{d}_{p}<0.0$ I vs all other groups. 
There is increased prevalence of obesity in persons with schizophrenia in the absence of treatment with antipsychotic drugs (Mukherjee et al, 1996; Ryan et al, 2003). Decreased nocturnal plasma melatonin concentrations have also been observed in persons with schizophrenia (Monteleone et al, 1992), although the possible role of melatonin in the pathophysiology of schizophrenia-associated weight gain has not, to our knowledge, been investigated. The current results thus suggest that olanzapine treatment may accentuate endocrine and associated metabolic disturbances characteristic of schizophrenia per se.

It was recently reported that 3-4 weeks olanzapine administration to eight male schizophrenia patients with predominantly negative symptoms did not significantly alter plasma melatonin levels, although the nocturnal rise appeared earlier (Mann et al, 2006). In the present rat study, nocturnal melatonin levels after 7 weeks of treatment were determined in single samples collected at the midpoint of the dark period so it is not possible to determine if the demonstrated olanzapine-induced decrease was due to reduced nocturnal peak amplitude or a similar phase shift in the time of the peak.

The mechanism of the melatonin effect on olanzapineinduced increases in body weight and visceral adiposity in rats is unclear. Locomotor activity was decreased by olanzapine, so decreased caloric expenditure may have contributed to the olanzapine-induced weight gain and increased adiposity. However, as addition of melatonin treatment did not alter this decreased locomotor activity, the melatonin-induced reversal of increased body weight and visceral adiposity during olanzapine treatment does not appear likewise to have been mediated by changes in locomotor activity.

Although olanzapine treatment induced an increase in eating during the first 2 weeks of treatment, food consumption during the subsequent 6 weeks of the trial was not significantly different among the treatment groups. Furthermore, food consumption by olanzapine + melatonin-treated rats was not different from that of rats treated with olanzapine alone, either initially or during the remainder of the study. This suggests that melatonin treatment did not decrease olanzapine-induced weight gain by suppressing eating. Melatonin replacement likewise did not alter food consumption in our previous studies of aging in which eating was rigorously characterized (WoldenHanson et al, 2000). However, it should be noted that the present study was not designed to thoroughly characterize eating, so the potential role of altered food consumption remains to be resolved.

Another candidate mechanism is suggested by evidence that melatonin may alter adipocyte fatty acid transport (Zurlo et al, 1990). This mechanism is consistent with findings in a recent human study of weight gain induced by 12 weeks of olanzapine treatment in unmedicated persons suffering from their first episode of acute schizophrenia (Graham et al, 2005a,b). In the human study, the most prominent metabolic effect of olanzapine was to decrease fatty acid oxidation.

Any substance added to drinking water potentially can decrease rat ingestive behaviors by being perceived as a novel taste or as toxic. However, in a previous study, we demonstrated that addition of melatonin to rats' drinking water at the same concentration as used in the present study did not alter preference relative to water that did not contain melatonin, did not alter volume of water consumed over $24 \mathrm{~h}$, did not alter water consumption in the subsequent $24 \mathrm{~h}$ after melatonin removal, and did not induce conditioned taste aversion (Wolden-Hanson et al, 2000). Thus, addition of melatonin to the water in this dosage was probably not detectable or perceived as aversive by the rats.

Although these results provide rationale for investigating whether olanzapine-induced endocrine and metabolic deregulation in humans can be blocked or attenuated by melatonin replacement therapy, these preliminary results require replication in longer studies to determine if the observed melatonin reduction of olanzapine-induced weight gain persists in a time course relevant to observations in humans. Olanzapine-induced weight gain has been observed to extend beyond 6 months in persons with schizophrenia (Wirshing et al, 1999). Future studies also should accurately measure effects of melatonin on eating and on endocrine parameters relevant to olanzapineinduced weight gain, such as plasma insulin and leptin. In our previous studies, melatonin reduction of rat insulin and leptin concentrations during aging were most clearly demonstrable under specific sampling conditions, that is, at the end of the light period after $9 \mathrm{~h}$ fasting for insulin, and during the dark period under non-fasting conditions for leptin (Puchalski et al, 2003). Other variability in responses could clearly also be owing to species, gender, and staterelated differences.

These results raise several other questions. Would similar results be observed in males, and is there a role for gonadal steroids in mediating metabolic responses to olanzapine? Would bolus administration of an appropriate dosage of melatonin in the evening be as effective as administration throughout the night, consistent with melatonin regulation of reproduction in at least some seasonal breeders (Stetson et al, 1986)? If so, evaluation and potential implementation of therapy based on melatonin effects on olanzapineinduced weight gain in humans would be more practical.

\section{ACKNOWLEDGEMENTS}

This work was supported by the Department of Veterans Affairs, Northwest Network VISN 20 Mental Illness Research, Education and Clinical Center (MIRECC) and NIH Grant AA013881. Olanzapine was provided by Eli Lilly \& Co., Indianapolis, IN.

\section{REFERENCES}

Allison DB, Mackell JA, McDonnell DD (2003). The impact of weight gain on quality of life among persons with schizophrenia. Psychiatr Serv 54: 565-567.

American Diabetes Association (2004). Consensus development conference on antipsychotic drugs and obesity in diabetes. Diabetes Care 27: 596-601.

Cagnacci A (1996). Melatonin in relation to physiology in adult humans. J Pineal Res 21: 200-213.

Fontaine KR, Heo M, Harrigan EP, Shear CL, Lakshminarayanan $\mathrm{M}$, Casey DE et al (2001). Estimating the consequences of 
antipsychotic induced weight gain on health and mortality rate. Psychiatry Res 101: 277-288.

Gao X-M, Sakai K, Tamminga CA (1998). Chronic olanzapine or sertindole treatment results in reduced oral chewing movements in rats compared to haloperidol. Neuropsychopharmacology 19: $428-433$

Glazer G (2001). Long-term pharmacotherapy of obesity 2000: a review of efficacy and safety. Arch Intern Med 161: 1814-1824.

Gothelf D, Falk B, Singer P, Kairi M, Phillip M, Zigel L et al (2002). Weight gain associated with increased food intake and low habitual activity levels in male adolescent schizophrenic inpatients treated with olanzapine. Am J Psychiatry 159: 10551057.

Graham KA, Gu H, Lieberman JA, Harp JB, Perkins DO (2005a). Double-blind, placebo-controlled investigation of amantadine for weight loss in subjects who gained weight with olanzapine. Am J Psychiatry 162: 1744-1746.

Graham KA, Perkins DO, Edwards LJ, Barrier Jr RC, Lieberman JA, Harp JB (2005b). Effect on olanzapine on body composition and energy expenditure in adults with first-episode psychosis. $A m \mathrm{~J}$ Psychiatry 162: 118-123.

Grad BR, Rozencwaig R (1993). The role of melatonin and serotonin in aging: update. Psychoneuroendocrinology 18: 283-295.

Henderson DC, Copeland PM, Daley TB, Borba CP, Cather C, Nguyen DD et al (2005). A double-blind, placebo-controlled trial of sibutramine for olanzapine-associated weight gain. Am J Psychiatry 162: 954-962.

Kinon BJ, Basson BR, Gilmore JA, Tollefson GD (2001). Long-term olanzapine treatment: weight change and weight-related health factors in schizophrenia. J Clin Psychiatry 62: 92-100.

Kissebah AH, Vydelingum N, Murray R, Evans DJ, Hartz AJ, Kalkhoff RK et al (1982). Relation of body fat distribution to metabolic complications of obesity. J Clin Endocrinol Metab 54: 254-260.

Krotkiewski M, Björntop P, Sjostrom L, Smith U (1983). Impact of obesity on metabolism in men and women: importance of regional adipose tissue distribution. J Clin Invest 72: 1150-1162.

Leucht S, Pischel-Wulz G, Abraham D, Kissling W (1999). Efficacy and extrapyramidal side-effects of the new antipsychotics olanzapine, quetiapine, risperidone and sertindole compared to conventional antipsychotics and placebo: a metaanalysis of randomized controlled trials. Schizophr Res 35: 51-68.

Lieberman JA, Stroup TS, McEvoy JP, Swartz MS, Rosenheck RA, Perkins DO et al (2005). Effectivenss of antipsychotic drugs in patients with chronic schizophrenia. $N$ Engl $J$ Med 353: 1209-1223.

Mann K, Rossbach W, Muller MJ, Muller-Siecheneder F, Pott T, Linde I et al (2006). Nocturnal hormone profiles in patients with schizophrenia treated with olanzapine. Psychoneuroendocrinology 31: 256-264.

Monteleone P, Maj M, Fusco M, Kemali D, Reiter RJ (1992). Depressed nocturnal plasma melatonin levels in drug-free paranoid schizophrenics. Schizophr Res 7: 77-84.

Mukherjee S, Decina P, Bocola V, Saracini F, Scapicchio PL (1996). Diabetes mellitus in schizophrenic patients. Compr Psychiatry 37: $68-73$.

Nelson RJ, Demas GE (1997). Role of melatonin in mediating seasonal energetic and immunologic adaptations. Brain Res Bull 44: 423-430.

Peschke D, Peschke E, Mess B (1987). Circannual rhythm and increase in body weight and food intake in the young wistar rat following pinealectomy and ganglioectomy. Neuroendocrinol Lett 9: 321-327.

Puchalski SS, Green JN, Rasmussen DD (2003). Melatonin effect on rat body weight regulation in response to high fat diet at middle age. Endocrine 21: 163-167.

Rasmussen DD, Boldt BM, Wilkinson CW, Yellon SM, Matsumoto AM (1999). Daily melatonin administration at middle age suppresses male rat visceral fat, plasma leptin, and plasma insulin to youthful levels. Endocrinology 140: 1009-1012.

Rasmussen DD, Mitton DR, Larsen SA, Yellon SM (2001). Agingdependent changes in the effect of daily melatonin supplementation on rat metabolic and behavioral responses. J Pineal Res 31 : 89-94.

Ryan MC, Collins P, Thakore JH (2003). Impaired fasting glucose tolerance in first-episode, drug-naïve patients with schizophrenia. Am J Psychiatry 160: 284-289.

Stein MB, Kline NE, Matloff JL (2002). Adjunctive olanzapine for SSRI-resistant combat-related PTSD: a double-blind, placebocontrolled study. Am J Psychiatry 159: 1777-1779.

Stetson MH, Sarafidis E, Rollag MD (1986). Sensitivity of adult male Djungarian hamsters (Phodopus sungonus suugorus) to melatonin injections through the day: effects on the reproductive system and the pineal. Biol Reprod 35: 618-623.

Wirshing DA, Wirshing WC, Kysar L, Berisford MA, Goldstein D, Pashdag J et al (1999). Novel antipsychotics: comparison of weight gain liabilities. J Clin Psychiatry 60: 358-363.

Wolden-Hanson T, Mitton DR, McCants RL, Yellon SM, Wilkinson CW, Matsumoto AM et al (2000). Daily melatonin administration to middle-aged male rats suppresses body weight, intraabdominal adiposity, and plasma leptin and insulin independent of food intake and total body fat. Endocrinology 141: 487-497.

Zurlo F, Lillioja S, Esposito-Del Puente A, Nyomba BL, Raz I, Saad MF et al (1990). Low ratio of fat to carbohydrate oxidation as a predictor of body weight gain: study of 24th RQ. Am J Physiol 259: E650-E657. 